\title{
Cryptocurrency: regulatory challenges, entrepreneurship and innovation impact
}

\author{
Alexandra M. Pogosyan, \\ Department of Management \\ Financial University under the Government of the Russian Federation \\ Moscow, Russia \\ pogosyan-alex@mail.ru
}

Abstract. It can be argued that cryptocurrencies are based on blockchain technology and have a wide range of advantages for customers. It has become a way of sharing value, investing or even earning money. Unfortunately, cryptocurrency is currently used for money laundering and financing terrorism. Governments of many countries are faced with the range of issues that need to be resolved, such as determining the essence of cryptocurrency, tracking operations and activities of cryptocurrency exchanges, taxation and many others. Moreover, there is a variety of challenges for the market, cryptocurrencies volatility in particular, which is necessary to consider.

Keywords: cryptocurrencies, cryptocurrency features, cryptocurrency exchanges, volatility, stablecoin, regulation.

\section{INTRODUCTION}

Since the concept of blockchains was proposed in 2008, digital currencies have been issued by online platformers as cryptocurrencies and traded online. Total market capitalization of cryptocurrencies has reached 247 billion dollars the following month [3].

Bitcoin is not only the first, but also the most common cryptocurrency today, which accounts for more than $65 \%$ of the capitalization of all cryptocurrencies in the world [2]. Other cryptocurrencies known as altcoins (Tether, Litecoin, Stellar, etc.) are developing nowadays exceeding the quantity mark of 5000 [7]. Altcoins combine common architectural principles and software solutions. In 2009, Bitcoin [10] introduced a new notion of decentralized cryptocurrency and trustless transaction processing and was followed by Ethereum [13], which introduced generalized scripting functionality, allowing smart contracts that execute algorithmically in a verifiable and somewhat trustless manner. Cryptocurrencies promise notions of cryptographic security, privacy, incentive alignment, digital usability, and open accessibility while removing most facets of counterparty risk.

\section{MAIN BODY}

Considering the main cryptocurrency features, it can be argued that:

- cryptocurrencies are decentralized, they do not have a single issuer, a centralized authority or controls;

- the cryptocurrency wallet holder can conduct transactions, that is, he/she can pay with cryptocurrency for goods and services on the Internet, as well as exchange them for other cryptocurrencies or fiat money;

- with no issuer, cryptocurrencies are not backed. Their exchange rate and value are extremely volatile and are supported by the participants of cryptocurrency activities themselves.

From the perspective of online payment systems, several studies have regarded blockchains as decentralized payment systems (DPSs) [11; 14]. DPSs studies are focused because not only is it easy to pay and remit overseas at lower cost, but DPSs also have the possibility to trace all transactions so that monetary policy is carried out in an algorithmic, automatic, and nondiscretionary way [9]. 


\section{Considering Cryptocurrency Regulation the following can ce stated.}

There is no single global definition of the term crypto- or virtual currency. In 2012, the European Central Bank (ECB) defined virtual currency as «a type of unregulated, digital money, which is issued and usually controlled by its developers, and used and accepted among the members of a specific virtual community» [12].

In 2014, The European Banking Authority (EBA) defined cryptocurrency as a "digital representation of value that is neither issued by a central bank or a public authority, nor necessarily attached to a fiat currency, but is accepted by natural or legal persons as a means of payment and can be transferred, stored or traded electronically" [4].

Financial Action Task Force on Money Laundering (FATF) gave the following definition on cryptocurrency: "the digital representation of value that can be digitally traded and functions as: (i) a medium of exchange; and/or (ii) a unit of account; and/or (iii) a store of value, but does not have legal tender status (i.e., when tendered to a creditor, is a valid and legal offer of payment) in any jurisdiction. It is not issued nor guaranteed by any jurisdiction, and fulfils the above functions only by agreement within the community of users of the virtual currency" [5].

In order to provide for a common regulatory approach through the fifth Anti Money Laundering Directive (MLD), the European Union decided to adopt a definition of virtual currency deriving from the FATF's guidance. According to MLD, a virtual currency is defined as a digital representation of value that is not issued or guaranteed by a central bank or a public authority, is not necessarily attached to a legally established currency, and does not possess a legal status of currency or money, but is accepted by natural or legal persons, as a means of exchange, and which can be transferred, stored and traded electronically. Given the broad nature of this definition, it is likely that, in practice, most forms of virtual currencies and other transferable cryptographic coins or tokens (as we know them today) fall within the scope of MLD.

With regard to the regulation in Russian Federation, cryptocurrency cannot be attributed to electronic money, as follows from Federal Law dated June 27, 2011 N 161 - FZ "On the National Payment System", since cryptocurrency is neither prepaid, nor a currency unit of the respective nations. Cryptocurrencies are not recognized in bank accounts or bank deposits according to Art. 1 of the Federal Law dated December 10, 2003 N 173 - FZ "On Currency Regulation and Currency Control:. As provided by paragraph 2, Art. 1 of the Federal Law "On Currency Regulation and Currency Control", cryptocurrency is not refereed to as foreign currency and does not fall under the objects of civil rights listed in Art. 128 of the Civil Code of the Russian Federation, since it is not an item (commodity), cash or non-cash, uncertificated securities or property rights and, therefore, refers to other property under Art. 128 of the Civil Code.

Cryptocurrencies face difficult technological, usability, and regulatory challenges to be successful in the long-term.

Many cryptocurrency systems develop different approaches to solve these problems. The value of these systems depends on network effects: value changes in a nonlinear way as new participants join. In concrete terms, the more people who use the system, the more likely it can be used to fulfill a given real world transaction [8]. The success of a cryptocurrency relies on a mass of agents - e.g., consumers, businesses, and/or financial institutions - adopting the system for economic transactions and value storage. Which systems will achieve this adoption is highly uncertainty, and so current cryptocurrency positions are very speculative bets on new technology.

Further, cryptocurrency markets face limited liquidity and market manipulation. In addition, the decentralized control and privacy features of cryptocurrencies can be at odds with desires of governments, which introduces further uncertainty around attempted interventions.

These uncertainties drive price volatility, which feeds back into fundamental usability problems. It makes cryptocurrencies unusable as short-term stores of value and means of payment, which increases the barriers to adoption. Indeed, today we see that most cryptocurrency transactions represent speculative investment as opposed to typical economic activity. 


\section{Stablecoin}

With the aim of stabilization, stablecoin is proposed to control the exchange rate between cryptocurrency and fiat currency. A stablecoin is a cryptocurrency with an economic structure built on top of blockchain that aims to stabilize the trading price.

In fact, several platformers have implemented stabilization mechanisms by issuing stablecoins. According to Hassani et al. [6], who investigate blockchain from the banker's perspective, stablecoin is defined as "basically a digital token that will have low price volatility as a result of being pegged to some underlying fiat currency, thereby acting as a store of value, a medium of exchange and unit of accounting for blockchain payments. The "stable" in "stablecoin" is surely about price volatility; however, it is too narrow to define it as "a result of being pegged to some underlying fiat currency". Hassani et al. [6] premise stablecoins Market capitalization is calculated by multiplying the market price per coin by the total amount of coins in circulation.

Stablecoins can be divided into four collateral types - fiat, crypto, commodity, and non-collateralized - according to the literature of Zhang et al. [15] and conclude that the non-collateralized stablecoin is the only way for a stabilized price, decentralization, and nondiscretionary digital currency. Second, we divide noncollateralized stablecoins into two layers (protocol and application) and show that non-collateralized stablecoins on the application layer is the simplest approach for implementation [9].

Different types of stablecoin initiatives can be identified in accordance with the criteria that characterize crypto-assets. These criteria are: (i) the existence/absence of an issuer that is responsible for satisfying any attached claim; (ii) the decentralization/centralization of responsibilities over the stablecoin initiative; and (iii) what underpins the value of a stablecoin and its stability in the currency of reference.

The stabilization mechanism at the core of a stablecoin initiative is crucial to determining whether the units issued can maintain a stable value or not. Different stabilization mechanisms may either require the intervention of accountable institutions, in the role of issuer and custodian, or delegate these tasks to stablecoin users [1].

\section{Cryptocurrency exhanges}

Despite the prolonged decline of cryptocurrencies in the first half of 2018, the number of crypto enthusiasts and crypto businessmen continues to grow steadily. Cryptocurrency exchanges allow them to change different cryptocurrencies to one another (Classic cryptocurrency exchanges) or traditional type funds (Fiat exchange). The latter allows to exchange fiat currency to crypto currencies and vice versa. Licensing is mandatory for the legitimate functioning of such an exchange, licenses can be different, namely:

- specialized crypto-licenses that are specifically introduced by the competent public authorities to regulate cryptocurrency exchange operations (Japan or Estonia);

- traditional licenses that regulate financial transactions and indirectly cover cryptocurrency transactions (the United States, Hong Kong).

As a rule, the crypto exchange in its activities must necessarily:

- $\quad$ conduct complex risk analysis procedures (due diligence);

- conduct regular staff training;

- draw up internal acts that will be aimed at minimizing the risks of money laundering;

- accounting,

- reporting suspicious transactions and regularly notifying the competent authorities about such transactions.

Considering licensing of the cryptocurrency exchange in Singapore, it is one of the few jurisdictions where the activity of cryptocurrency exchanges is directly regulated by current legislation at the moment. During 2014-2018 the national regulator (MAS) issued clarifications expressing its position regarding cryptocurrencies, ICOs, and licensing of the cryptocurrency exchange. Despite the mentioned fact, in 2019 a new legislative act was adopted. The Payment Service Act obliges 
companies engaged in exchanging cryptocurrency for other cryptocurrencies or for fiat funds to obtain a Small Payment Institution or Major Payment Institution License.

Cryptocurrency exchanges are intermediaries in the exchange of virtual currencies and Fiat money. It should also be taken into account that if a cryptocurrency exchange starts acting as an intermediary for the purchase and sale of tokens, it become subjected to securities legislation, it will be necessary to obtain a license for a full-fledged stock exchange.

In addition to the volatility of the cryptocurrency exchange rate, which decreases with the appearance of stablecoins, there are other problems as:

- Placement: VCs offer the ability to open a significant number of anonymous or pseudonymous wallets, at no or very low cost, which is a low-risk method of rapidly placing proceeds of illicit activity.

- Layering: cryptocurrencies enable the source of funds to be obfuscated by means of multiple transfers from wallet to wallet and/or their conversion into different types of cryptocurrencies across borders. This allows for an easy layering without significant cost or risk, it being understood that recent technological developments such as "atomic swaps" may even further facilitate the misuse of cryptocurrencies.

- Integration: the use of cryptocurrency to acquire goods or services, either directly or through the conversion of the cryptocurrencies into fiat currency, is facilitated by the ever-increasing list of goods and services for which payment in cryptocurrencies is accepted, as well as the entry into the cryptocurrency markets of institutional players both for investment and trading (speculation) purposes, providing substantial liquidity in the cryptocurrency markets and thereby potentially facilitating large-scale integration by abusing unsuspecting institution actors/investors.

- Cryptocurrency could be used for money laundering and financing of terrorism purposes. Although the global volumes and estimates are relatively low, Europol has estimated in 2017 that $3-4 \%$ of Europe's crime proceeds were laundered through cryptocurrencies - the proportion will likely continue to increase rapidly due to the rate of adoption of cryptocurrencies, including by institutional investors and FIs.

Given the trans-jurisdictional or borderless nature of the cryptocurrency phenomenon, major institutions at the international level have all focused on and issued reports addressing cryptocurrencies and the risks associated with them, including laundering and financing of terrorism risks.

The rapid development, increased functionality, and growing adoption of new technologies and related payment products and services globally continue to pose significant challenges for regulators and private sector institutions in ensuring that these technologies are not misused for money laundering and financing of terrorism purposes.

FATF and EBA, in particular, have issued recommendations in this context, concluding that cryptocurrency exchange platforms allowing the conversion of cryptocurrencies into fiat money and vice versa are of particular relevance and must be brought within the scope of the respective national anti-money laundering and counter-financing of terrorism (AML/CFT) frameworks. Cryptocurrency exchanges specifically must conduct a procedure for identifying token issuers before placing the corresponding tokens on their platform. As a rule, the burden of paying all related financial costs falls on token issuers.

Some of the amendments adopted in March 2019 are taking effect in 2020 and oblige all market participants to comply with strict rules regarding data security, storage of funds, margin trading and attracting new customers. In addition, license fees were increased.

\section{CONCLUSION}

As decentralized, convertible cryptography-based virtual currencies and related payment systems are gaining momentum, regulators and financial institutions around the world are recog- 
nizing that virtual currencies and the underlying consensus protocols likely represent the future for payment systems.

Despite the advantages, most online payment systems have not employed a cryptocurrency as an alternative to a fiat currency. In fact, in eight of the largest cryptocurrency markets (US, UK, Germany, Brazil, Japan, South Korea, China and India), current awareness of cryptocurrency is $74 \%$ on average, while its ownership is on average $7 \%$. Most of the owners of online shops do not choose DPSs due to the high price volatility of cryptocurrency.

Introduction of cryptocurrency into modern society is massive and the governments should take more loyal position regarding the legal status of cryptocurrency. The adoption of special legislative regulation of cryptocurrency in Russia will help to maintain a balance between technological innovations and market. In this regard, it is also necessary to develop special rules and recommendations to improve investigation process of criminal offenses. Russian Federation could implement best international legal practices to stop illegal cryptocurrency transactions.

\section{REFERENCES}

[1]. Bullmann D., Klemm J., Pinna A. Occasional Paper Series In search for stability in crypto-assets: are stablecoins the solution?, Occasional Paper Series in European Central Bank, 2019, no 230.

[2]. Coin Market Cap. Available at: https://coinmarketcap.com/charts/\#dominance-percentage.

[3]. CoinMarketCap Global Charts. Available at: https://coinmarketcap.com.

[4]. European Banking Authority (EBA) Opinion on virtual currencies, 4 July 2014.

[5]. FATF REPORT Virtual Currencies Key Definitions and Potential AML/CFT Risks. Available at: https://www.fatf-gafi.org/media/fatf/documents/reports/Virtual-currency-key-definitions-and-potential-aml-cft-risks.pdf.

[6]. Hassani H., Huang X., SilvaE. Banking with blockchain-ed big data. Journal of Management Analytics, vol. 5, n 4, 2018.

[7]. IcoBENCH. Available at: https://icobench.com/.

[8]. Klages-Mundt A., Minca A. (In) Stability for the Blockchain: Deleveraging Spirals and Stablecoin Attacks. [S.1.,] 2019.

[9]. Mita M., Ito K., Ohsawa S., Tanaka H. What is Stablecoin?: A Survey on Price Stabilization Mechanisms for Decentralized Payment Systems. [S.1.,] 2019.

[10]. Nakamoto S. Bitcoin: a peer-to-peer electronic cash system. Available at: https://bitcoin. org/bitcoin.pdf.

[11]. Decentralized Payment Systems: Principles and Design, eds. G. Fanti and P. Viswanath), The Distributed Technology Research Foundation. 2019.

[12]. Virtual currency schemes, October 2012, European Central Bank. Available at: http://www. ecb.europa.eu.

[13]. Wood G. Ethereum: a secure decentralised generalised transaction ledger. Ethereum Yellow Paper. Available at: https://ethereum.github.io/yellowpaper/paper.pdf.

[14]. Xu A., Li M., Huang X., Xue N. A Blockchain Based Micro Payment System for Smart Devices. Suzhou: 1st Conference on Emerging Topics in Interactive Systems. [S.1.], 2016.

[15]. Zhang A. R., Raveenthiran A., Mukai J., et al. The Regulation Paradox of Initial Coin Offerings: A Case Study Approach. Schulich School of Business, York University. SSRN Electronic Journal, 2018. 